\title{
Simple data approximation for computer and controller-aided devices
}

\author{
Uldis Žaimis, Dzintars Tomsons, Valdis Priedols \\ Liepaja University, Faculty of Science and Engineering \\ Institute of Science and Innovative Technologies
}

\section{INTRODUCTION}

Development of mechatronic systems involves finding an optimal balance between the basic mechanical structure, sensor and actuator implementation, automatic information processing and overall control. Mechatronic systems are characterized by a combination of basic mechanical devices with a processing unit monitoring and controlling it via number of actuators and sensors. Therefore sensors are significant in the process of providing usable output to microcontrollers. Wide range of sensors are available for constructing mechatronic systems. Sensors can be divided into two big groups: Active and Passive. Other type of classification is by the means of detection used in the sensor. Some of the means are electric, chemical, radioactive etc. Various types of sensors are classified by their measuring objectives for example light sensors, temperature sensors, flow sensors etc.

\section{MATERIALS AND METHODS}

In the process of constructing a mechatronic system a proper setup and signal processing must be provided. There exist certain problems with several sensors, therefore sometimes additional circuits for signal conditioning are made to linearize the output with hardware, but some researchers and developers try to linearize the signal using software.

In modern manufacturing equipment very complex systems of devices and sensors are made therefore, they must function correctly because they are the main control parameters. It is particularly important that such control parameters that bring about a correct actual behavior in relation to the reference behavior of such a system are available as a function of time. This means that the parameters must be such that the actual behavior of the system corresponds as closely as possible to the reference behavior. Some examples of such systems are:

- Robot arms, which move a tool, such as a laser or burr removing tool, for example, which is to be guided along a particulary contour line of a workpiece.

- Heating systems which are intended to impart a particulary temperature profile to a workpiece.

The input data of sensors is crucial for mechatronic systems.

A large part of the system is equipped with sensors that read the most important parameters - location coordinates, altitude, compass readings, distance to the barrier (for robots and unmanned aerial vehicles), temperature (heaters and coolers), lighting, etc.

Often, some types of sensors give floating data, processing which, a computer or controller acting under an algorithm develops non-physical, inexecutable commands for the final control elements. This results in an increasing load of engines, heating elements, and other actuators, as well as inappropriately increasing energy consumption.

The well-known PID algorithm and numerical approximation with built-in MatLab or MATCAD functions does not provide a solution for autonomous systems with controllers that have limited memory and speed of operation.

\section{RESULTS}

New methods that approximate sensor data and are applicable to both analogue and PWM (Pulse-Width-Modulation)-controlled devices have been developed in the paper.

The first proposed - derivative - method relates to the restriction of the function direction coefficient module. The second method - the growth bisection method enables smooth sensor data to be obtained.

The derivation method is based on limitation of the maximum function increment to a specified level. The growth bisection (proportional) method is based on comparison of the increment module with the increment in the previous step and its proportional decrease by multiplying by a predefined constant. Both methods take up some lines in the control program code, and most mechatronic equipment is capable of real-time operation.

\section{CONCLUSIO}

Dynamic data background connection allows to obtain a self-learning system adapting to the nature of incoming data - a higher number of data will be used in case of minor changes; in contrast, only the last data saved will be used for a rapid change. A system response delay is negligible.

\section{KEYWORDS}

Data, approximation, algorithm, controller-aided systems 